\title{
From Pioneers to Team Players: TGA Transcription Factors Provide a Molecular Link Between Different Stress Pathways
}

\author{
Christiane Gatz \\ Georg-August-University of Göttingen (GAU), Albrecht-von-Haller-Institute for Plant Sciences, Julia-Lermontowa-Weg 3, \\ 37077 Göttingen, Germany
}

Submitted 3 April 2012. Accepted 11 September 2012.

\begin{abstract}
The plant immune system encompasses an arsenal of defense genes that is activated upon recognition of a pathogen. Appropriate adjustment of gene expression is mediated by multiple interconnected signal transduction cascades that finally control the activity of transcription factors. These sequence-specific DNA-binding proteins act at the interface between the DNA and the regulatory protein network. In 1989, tobacco TGA1a was cloned as the first plant transcription factor. Since then, multiple studies have shown that members of the TGA family play important roles in defense responses against biotrophic and necrotrophic pathogens and against chemical stress. Here, we review 22 years of research on TGA factors which have yielded both consistent and conflicting results.
\end{abstract}

\section{The pioneer: Tobacco TGA1a.}

After the discovery of the flow of genetic information from DNA to RNA to protein in the middle of the last century, the question how this fundamental process is controlled was of major interest in molecular biology. Initiation of transcription is an essential step in gene expression and the prokarytic lac operon constituted a paradigm for how sequence-specific DNA binding proteins can control the activity of a promoter. While the sequence of the lacI gene was released in 1978 (Farabaugh 1978), the sequences of the first yeast transcription factors (GAL4, GCN4) became known in 1984 (Laughon and Gesteland 1984; Penn et al. 1984). Whereas these were cloned by complementation of the corresponding mutants, isolation of regulatory factors from higher eukaryotes was more difficult. Nevertheless, sequences of the first human transcription factors (e.g., glucocorticoid receptor, cJUN, and SP1) became known between 1985 and 1988 (Hollenberg et al. 1985; Kadonaga et al 1987; Ryder et al. 1988). Isolation strategies that were based on the specific DNA-binding activities (Singh et al. 1988) were instrumental for the cloning of the first plant transcription factor in 1989 (Katagiri et al. 1989).

Thus, the characterization of essential cis elements in the plant viral Cauliflower mosaic virus (CaMV) $35 \mathrm{~S}$ promoter was an important prerequisite (Lam et al. 1989). At that time,

Corresponding author: C. Gatz: E-mail: cgatz@uni-goettingen.de

(C) 2013 The American Phytopathological Society viral promoters that were efficiently recognized by the plant transcriptional machinery were believed to bind host transcription factors with high efficiency. Two functionally important TGACG motifs were identified between base pair positions -63 and -83 . This sequence was subsequently called activation sequence-1 (as-1). In tobacco nuclear extracts, an as-1-binding protein complex was identified that was named activation sequence factor-1 (ASF-1). In order to clone as-1-binding proteins, a tobacco cDNA expression library was screened with radioactively labeled oligonucleotides encoding multiple TGA CG motifs. In 1989, this strategy culminated in the first report describing the amino-acid sequence of TGA1a (Katagiri et al. 1989).

The primary structure of TGA1a revealed homology to the basic domain leucine zipper (bZIP) motif found in transcription factors from other kingdoms (e.g., human cJUN and CREB, yeast GCN4). In TGA1a, the basic DNA-binding domain consists of 20 amino acids with six arginine and four lysine residues. The following 21 amino acids contain three leucine residues in every seventh position. Such a bZIP element adopts an uninterrupted alpha-helical conformation with the leucine side chains forming a hydrophobic interface for dimerization (Ellenberger et al. 1992; Landschulz et al. 1988). Accordingly, bZIP transcription factors bind as dimers to palindromic sequences with each DNA-binding domain recognizing one half site. Sequence alignment of TGA1a with the ten Arabidopsis TGA factors reveals a conserved domain structure that extends beyond the bZIP element (Fig. 1). The bZIP region is preceded by a variable $\mathrm{N}$-terminal domain ranging from 45 to 187 amino acids. In contrast, the 244 to 263 amino-acid domain following the bZIP element is more conserved. Two glutamine-rich sequences (Q1, eight glutamines in 29 residues and Q2, six glutamines in 25 residues) were already highlighted in the first publication on TGA1a (Katagiri et al. 1989) and are found in all members of the Arabidopsis TGA family.

In the years following the discovery of TGA1a, several in vitro studies documented that TGA factors bind to variants of the palindromic sequence TGAC/GTCA, the minimal requirement being an intact TGACG motif (Izawa et al. 1993; Katagiri et al. 1992; Schindler et al. 1992). The activation domain of TGA1a was mapped to the 87-amino acids long $\mathrm{N}$ terminus (Neuhaus et al. 1994). The basic DNA-binding domain encodes a bipartite nuclear localization domain (van der Krol and Chua 1991). Weak dimerization due to the short zipper domain (only three leucine residues as compared with five in cJUN) is compensated by a C-terminal dimerization domain, which at 
the same time interferes with formation of mixed dimers between different TGA proteins (Katagiri et al. 1992).

In parallel to studies focusing on the properties of the TGA1a protein, different laboratories aimed to unravel their function in planta. Expression of a potato TGA factor with an altered DNA-binding domain (Lex-PG13) in tobacco led to reduced ASF-1 activity, indicating that ASF-1 consists of TGA factors that form inactive dimers with Lex-PG13 through the ZIP region (Rieping et al. 1994). Subsequent studies found that ASF-1 consists predominantly of TGA2.2 and contains only a small amount of TGA1a (Kegler et al. 2004; Niggeweg et al. 2000). Interfering with ASF-1 activity correlated with compromised expression of an as-1-driven reporter construct (Rieping et al. 1994). This result provided the first loss-offunction evidence that TGA factors are involved in activating gene expression from the as- 1 element.

The presence of TGACG motifs in the promoters of several plant glutathione S-transferase genes (Ellis et al. 1993; van der Zaal et al. 1996) gave rise to the hypothesis that TGA proteins might activate plant stress-responsive genes. Chimeric promoters consisting of either one or multiple $a s$-1- or $a s$-1-like elements upstream of a minimal CaMV $35 S$ promoter are responsive to multiple chemical stress cues including the plant defense hormones salicylic acid (SA) and jasmonic acid (JA), the auxin-related herbicide 2,4-dichlorophenoxyacetic acid (2,4-D) and $\mathrm{H}_{2} \mathrm{O}_{2}$ (Liu and Lam 1994; Qin et al. 1994; Xiang et al. 1996). Chemicals structurally related to SA or 2,4-D (3hydroxybenzoic acid and 2,6-D, respectively) do not induce TGA-mediated gene expression (Niggeweg et al. 2000). Still, the coding regions of as-1-containing target genes and the high concentrations of chemicals that must be used for induction (Pascuzzi et al. 1998) argue for a role of TGA factors in the detoxification of these compounds. Moreover, as-1-driven promoters are active in root tips (Benfey et al. 1990; van der Zaal et al. 1991), which correlates with the expression domain of TGAla and the related gene PG13 (Klinedinst et al. 2000). It was discussed that the high abundance of TGA factors in meristems would serve to activate genes that counteract the oxidative stress occurring in rapidly dividing cells (Zhang and Singh 1994).

Since transcript levels of TGAla were unaffected under inducing conditions, it was hypothesized that TGA activity is regulated at the posttranscriptional level (Liu and Lam 1994). Electrophoretic mobility shift analyses (EMSA) of ASF-1 or recombinant TGA1a in the presence of tobacco extracts suggested that inhibitory proteins would interfere with DNA binding of TGA factors in the noninduced state. Stress-induced phosphorylation of either TGA factors or these inhibitory proteins would lead to the dissociation from this inhibitory complex (Johnson et al. 2001; Jupin and Chua 1996; Pascuzzi et al. 1998; Stange et al. 1997). Since as-1-driven transcription and ASF-1 binding activity to $a s-1$ are induced by the protein synthesis inhibitor cycloheximide (Liu and Lam 1994), it was speculated that the hypothetical repressive proteins are subject to a rapid turnover. Up to now, these proteins have not been identified.

After the advent of Arabidopsis thaliana as one of the prime model systems for plant molecular biology, analysis of the TGA family was mainly performed in Arabidopsis. The release of the Arabidopsis genome sequence unraveled ten members of the TGA family falling into five clades (Jakoby et al. 2002). Clade I comprises TGA1 (At5g65210) and TGA4 (At5g10030), which are most similar to tobacco TGA1a (Fig. 1). Clade II consists of three closely related factors TGA2 (At5g06950), TGA5 (At5g06960), and TGA6 (At3g12250), which contain shorter $\mathrm{N}$ termini than the other TGA factors. TGA3 (At1g22070) and TGA7 (At1g77920) constitute clade III. PERIANTHIA (At1g68640) and TGA9 and TGA10 (At1g08320, At5g06839) form the remaining two clades, respectively.

\section{From a pioneer with uncertain functions to central players of the plant immune system: Clade II TGA factors are essential regulators of systemic acquired resistance.}

In 1998 and 1999, two independent lines of evidence pointed at TGA factors being essential regulators of the SA-induced defense network. First, a TGACG motif (LS7) was found to be

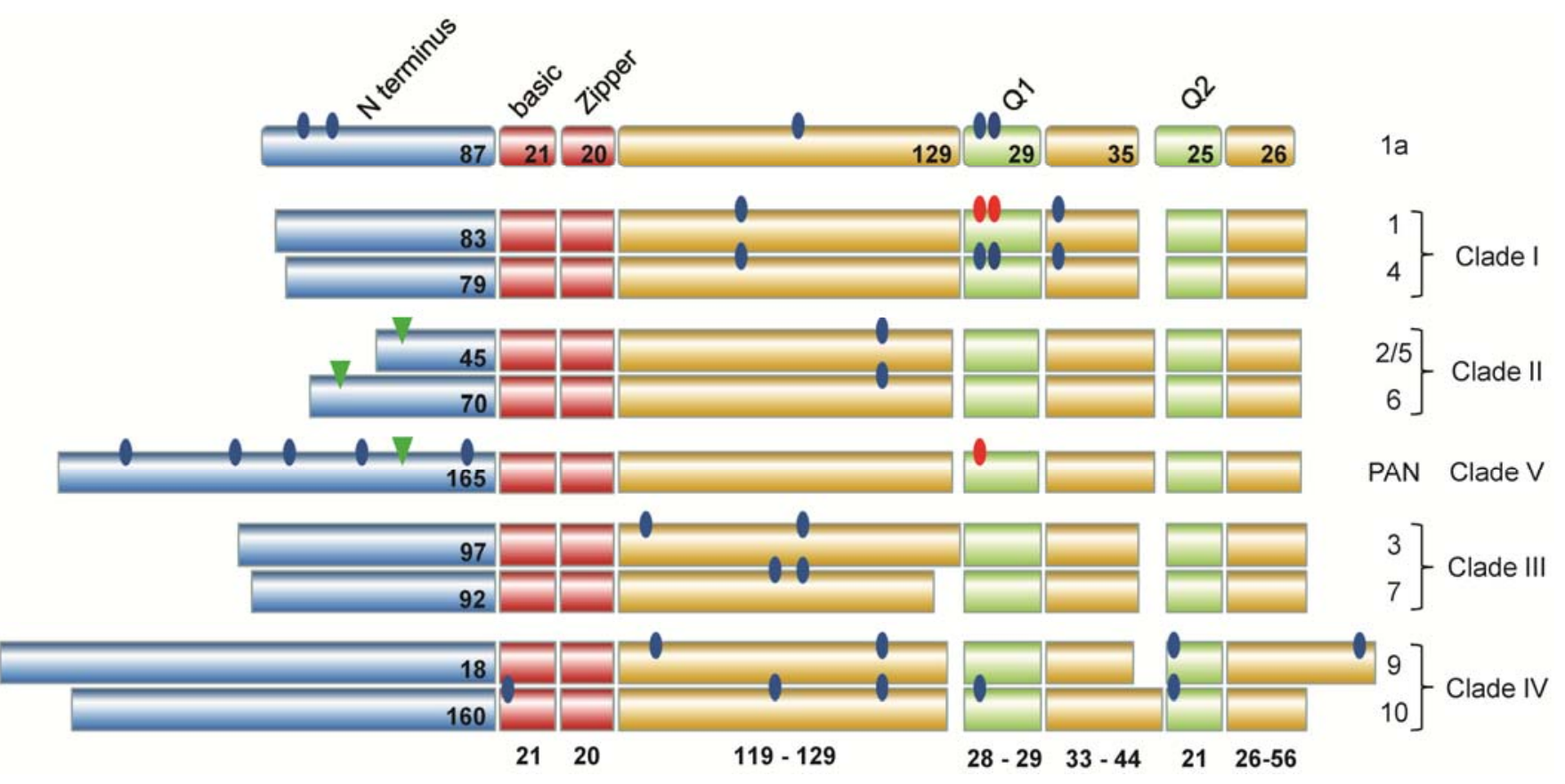

Fig. 1. Primary structure of TGA transcription factors. The founding member TGA1a from tobacco and the 10 Arabidopsis TGA factors are displayed. Different domains are indicated by different colors. Glutamine-rich domains are denoted Q1 and Q2. Numbers in the domains of TGA1a and in the N termini indicate the number of amino-acid residues. Numbers below the domains indicate the range of the size of the individual domains in different TGA factors. Balls mark the position of cysteine residues; functionally important cysteines are marked in red. Green triangles denote a STDxDT motif that can be phosphorylated. 
stringently required for the activation of the PATHOGENESISRELATED-1 (PR-1) gene in Arabidopsis seedlings treated with SA or its analogue 2,6-dichloroisonicotinic acid (INA) (Lebel et al. 1998). Second, clade II TGA factors (TGA2, TGA5, TGA6) were identified as interaction partners of the ankyrin repeat protein NPR1 (NONEXPRESSOR OF PR GENES 1 [Zhang et al. 1999]). NPR1 is required for expression of $P R-1$ and for the establishment of the SA-dependent defense response called systemic acquired resistance (SAR) (Zhang et al. 2003). The finding that TGA factors act at the interface between the non-DNA-binding protein NPR1 and the $P R-1$ promoter established them as part of a hormone-controlled signaling cascade that is efficient against biotrophic pathogens. Since clade I TGA factors did not interact with NPR1 in the yeast twohybrid system (Zhou et al. 2000) and since clade II TGA factors constitute the main component of the Arabidopsis ASF-1 complex in leaf nuclear extracts (Lam and Lam 1995), subsequent analyses focused on TGA2, TGA5, and TGA6. Due to the short genomic distance between TGA2 (At5g06950) and TGA5 (At5g06960), reverse genetics exploiting knock-out mutants was hampered for a time. Therefore, expression of a dominant negative TGA protein in transgenic plants was chosen as an alternative strategy to prove that TGA factors and NPR1 act in the same pathway. The C-terminal domain of TGA2, which interacts with NPR1 but cannot bind to DNA, interferes with INA-induced $P R-1$ expression, most likely by sequestering NPR1 into an inactive complex. Like nprl, plants expressing this protein show an enhanced disease susceptibility phenotype (Fan and Dong 2002). A fast neutron deletion mutagenesisbased reverse genetics system was deployed to isolate the tga2-1 tga5-1 deletion line ( $\mathrm{Li}$ et al. 2001). Crossing of tga2-1 tga5-1 with tga6-1 yielded a mutant that displays an nprl-like phenotype with respect to compromised SAR and lack of $P R-1$ induction after treatment with the SA analogue INA (Zhang et al. 2003). Although TGA2, TGA5, and TGA6 act redundantly with respect to SAR establishment, they exert different functions in the noninduced state. TGA2 suppresses basal levels of $P R-1$ expression whereas TGA6 functions as an activator if it is overexpressed (Kesarwani et al. 2007). The basal transcriptional activation observed in the tga2-1 tga5-1 tga6-1 mutant is due to the activity of TGA3, as revealed by analysis of the tga2-1 tga3-1 tga5-1 tga6-1 quadruple mutant (Kesarwani et al. 2007). Susceptibility against virulent pathogens is increased in $n p r 1$ but not in tga2-1 tga5-1 tga6-1, indicating that NPR1 interacts with other factors in basal resistance.

\section{An area of debate: Mechanistic aspects of the regulation of NPR1- and TGA-dependent gene expression under inducing and noninducing conditions.}

Whereas genetic data convincingly demonstrate that the NPR1-TGA interaction is required for SAR establishment, studies on mechanistic aspects have yielded conflicting results. According to the current model, the majority of NPR 1 is located as a multimer in the cytosol of unchallenged plant cells (Mou et al. 2003). As soon as SA levels increase, a redox change in the cell leads to the reduction of critical cysteine residues in NPR1. Subsequently, monomeric NPR1 translocates into the nucleus, in which it interacts with TGA factors (Fig. 2A). Early studies implicated NPR1 as an enhancer of the binding of TGA factors to the DNA. One line of evidence was based on a chimeric transcription factor containing the DNA-binding domain of yeast transcription factor GAL4 instead of the bZIP domain of TGA2 (Fan and Dong 2002). Cellular extracts obtained from uninduced seedlings expressing TGA2-GAL4 show lower binding activity to GAL4 motifs in EMSA than do cellular extracts from seedlings grown on INA. Importantly, no GAL4 binding activity to its recognition site was detected when the construct was expressed in the npr 1-2 mutant background. Consistently, Arabidopsis nuclear ASF-1 was reported to bind to a TGACG-containing DNA probe in an SA- and NPR1-dependent manner (Johnson et al. 2003). However, another study reported a constitutively enhanced ASF-1 activity when using cellular extracts from the nprl mutant for EMSA (Despres et al. 2000). Nevertheless, data published in the same study supported the idea that NPR1 would enhance the DNA binding of TGA proteins using
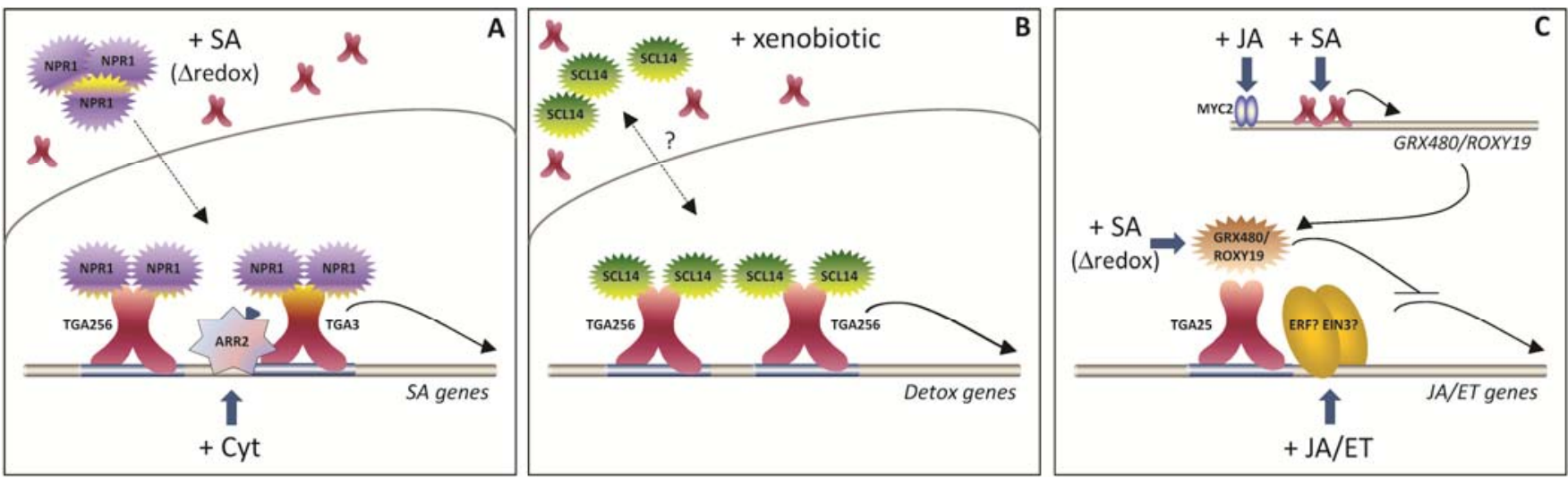

Fig. 2. Working models of TGA factors and their interacting proteins at promoters activated by different signal transduction cascades. A, Cytokinin-enhanced salicylic acid (SA)-induced expression of defense genes. Elevated SA levels lead to a change of the cellular redox state, resulting in the reduction of critical cysteines of NPR1 and translocation of the protein into the nucleus (Mou et al. 2003). TGA transcription factors TGA2, TGA3, TGA5, and TGA6 can recruit the transcriptional coactivator NPR1 to the promoters of SA-induced genes (like $P R-1$ ). In cytokinin-rich tissues, TGA3 recruits phosphorylated (blue triangle) response regulator ARR2, leading to hyperactivation of some SA genes (Choi et al. 2012). TGA2 and possibly TGA5 and TGA6 are located in the cytosol and the nucleus (Cheng et al. 2009). B, Xenobiotic-induced expression of detoxification genes. Class II TGA factors are required for the activation of xenobioticinducible genes like CYP81D11. The GRAS protein SCL14, which is activated by a yet-unknown mechanism, is required as a transcriptional coactivator (Fode et al. 2008). SCL14, TGA2, and possibly, TGA5 and TGA6 are located in the cytosol and the nucleus (Cheng et al. 2009). C, Jasmonic acid and ethylene (JA/ET)-induced expression of defense genes. Induction of JA/ET genes depends on class II TGA factors if JA and ET are applied at the same time. Under these conditions, JA-induced MYC2 dampens expression levels (Lorenzo et al. 2004), potentially by triggering the expression of GRX480/ROXY19, as deduced from the observations that ectopically expressed GRX480/ROXY19 suppresses PDF1.2 expression (Ndamukong et al. 2007) and that JA induction of GRX480/ROXY19 depends on MYC2 (Koster et al. 2012). If SA is applied, PDF1.2 expression is further down-regulated, potentially due to a higher repressive efficiency of GRX480/ROXY19 under reducing conditions. If TGA factors and MYC2 are missing (low GRX480/ROXY19 levels), wild-type PDF1.2 transcript levels are observed after JA/ET treatment, but the responsiveness to SA is abolished (Zander et al. 2010). 
in vitro translated proteins. The NPR1-TGA-DNA complex was not detected in EMSA, suggesting that NPR1 would serve to facilitate DNA binding of TGA factors rather than forming a stable complex. This interpretation is consistent with the finding that in vitro translated NPR 1 binds preferentially to recombinant TGA proteins that are not in their DNA binding-competent conformation (Johnson et al. 2008). In support of the above-mentioned results, chromatin immunoprecipitation studies provided evidence for SA-induced, NPR1-dependent recruitment of TGA factors to the $P R-1$ promoter (Johnson et al. 2003). While these data were obtained with antibodies against the $\mathrm{N}$ termini of TGA2 and TGA3, immunoprecipitation of a tagged TGA2 protein expressed under the control of the $C a M V$ $35 S$ promoter revealed constitutive binding of TGA2 to the $P R-1$ promoter region, independently of SA and NPR1 (Rochon et al. 2006). In vitro data suggest that the $\mathrm{N}$ terminus of TGA2 mediates multimerization of TGA2 at the $P R-1$ promoter; this multimer was proposed to dissociate upon association with NPR1 with TGA2 (Boyle et al. 2009). Assuming that the antibody against the $\mathrm{N}$ terminus of TGA2 might not recognize TGA2 in such a repressive complex, chromatin immunoprecipitation with this antibody might have failed to detect TGA2 at the inactive $P R-1$ promoter (Johnson et al. 2003). However several discrepancies have remained. First, chromatin immunoprecipitation experiments with the antibody against the tagged TGA2 should have yielded reduced TGA2 levels at the activated promoter. Second, the results of the EMSA experiments implicate induced recruitment of TGA2. Therefore, the question of whether TGA factors require NPR1 for binding to their target promoters or whether NPR1 leads to the dissociation of a large fraction of TGA2 at the activated promoter is not yet resolved. However, it is generally accepted that NPR1 functions as a transcriptional coactivator (Spoel et al. 2009), with SA potentially binding to its transcriptional activation domain (Wu et al. 2012).

The observation that the $P R-1$ promoter shows elevated basal expression levels in the tga2-1 tga5-1 tga6-1 mutant raised the question of whether TGA factors could also act in a negative way, at least on this promoter. Since mutation of the as-1-like element leads to enhanced $P R-1$ expression levels (Pape et al. 2010), it seems plausible that TGA factors can exert their repressive activity in cis. However, this would require at least some basal levels of promoter occupancy by TGA factors, as suggested in some studies (Boyle et al. 2009; Rochon et al. 2006). Based on the observation that $P R-1$ transcript levels are increased in the npr3 npr4 double mutant (Zhang et al. 2006), it has been suggested that NPR1-related proteins NPR3 and NPR4 might form a repressive unit by binding to clade II TGA factors in the absence of SA at the $P R-1$ promoter (Pape et al. 2010). Recent results have shown that NPR1 levels increase in the absence of NPR3 and NPR4 (Fu et al. 2012), which would also explain the elevated $P R-1$ transcript levels in npr3 npr4. This explanation can be reconciled with the finding that basal activity in the tga2-1 tga5-1 tga6-1 mutant depends on TGA3 (Kesarwani et al. 2007). However, when inferring molecular mechanisms from the analysis of $P R-1$ expression in different mutants, it has to be considered that the $P R-1$ promoter is directly and indirectly regulated by the NPR1/TGA complex. Even a $P R-1$ promoter derivative lacking the $a s$ - 1 -like element responds to INA in an NPR1-dependent manner (Pape et al. 2010). Presumably, SA-inducible WRKY transcription factors, many of them being under the direct control of the TGA/NPR1 complex (Wang et al. 2006), activate the promoter. The $P R-1$ promoter had originally been chosen for the analysis because it displays low background levels and high induced expression that is most likely due to direct and indirect effects of repressing and activating proteins.
Clade I TGA factors become interesting again: The redox state of Arabidopsis TGA1 is modulated by SA.

Since the redox state of the cell is altered after Pseudomonas infections or treatment with INA (Mou et al. 2003) and since TGA factors interact with glutaredoxins (GRX) (Li et al. 2009; Ndamukong et al. 2007; Zander et al. 2012), they appear to be likely targets of a redox modification process. Clade II TGA factors contain only one cysteine (Fig. 1), which may or may not be oxidized. However, Cys-260 and Cys-266 in the Q1 region of TGA1 form a disulfide bridge (Despres et al. 2003). These cysteines are conserved in TGA4 and tobacco TGA1a. When they are mutated to asparagine and serine, respectively, TGA1 can interact with NPR1 in a yeast two-hybrid system, which is not the case for the wild-type protein. The relevance of these data is supported by the observation that the disulfide bridge in TGA1 is reduced in SA-treated Arabidopsis leaf cells. Moreover, an in planta two-hybrid protein interaction system revealed that nuclear localized GAL4-NPR1 interacts with TGA2 but not with TGA1, unless SA is added (Despres et al. 2003; Rochon et al. 2006).

Although the biochemical data suggest a functional context of the SA-induced and redox-controlled TGA1-NPR1 interaction, Clade I TGA-mediated defense reactions are largely NPR1-independent (Shearer et al. 2012). The tgal-1 tga4-1 double mutant displays enhanced susceptibility to Pseudomonas syringae pv. maculicola ES4326 (Kesarwani et al. 2007) and Hyaloperonospora arabidopsidis Emwa1 (Shearer et al. 2012). However, TGA 1 and TGA4 control basal resistance independently from NPR1. Moreover, they are not involved in the establishment of SAR. Still, basal $P R-1$ transcript levels are enhanced in the tgal-1 tga4-1 mutant (Lindermayr et al. 2010). Surprisingly, these are not fully reverted to wild-type levels by a CaMV35S:TGA1 construct. Replacement of Cys260 and Cys-266 and two other cysteines by serine residues does not convincingly alter the low efficiency of the wild-type protein to suppress basal $P R-1$ transcript levels. Since $P R-1$ expression was only monitored under noninducing conditions, conclusions on the potential of these residues to function in an SA and NPR1-regulated process cannot be drawn. Neither wild-type TGA1 nor any of the mutated proteins complement the eds phenotype of the tgal-1 tga4-1 mutant.

\section{Connecting SA with cytokinin: Clade III TGA factors.}

Clade III encompasses TGA3 and TGA7. Like the tgal-1 tga4-1 double mutant, the $\operatorname{tga} 3-1$ mutant is more susceptible to Pseudomonas syringae pv. maculicola ES4326 (Kesarwani et al. 2007). Although TGA3 and NPR1 physically interact in the yeast two-hybrid system (Zhou et al. 2000), TGA3 acts partially independent from NPR1, as revealed by the higher susceptibility of the tga3-1 nprl-1 double mutant as compared with the respective single mutants.

The phytohormone cytokinin supports SA-mediated responses. Based on the observations the tga3-1 mutant fails to confer cytokinin-enhanced resistance against Pseudomonas syringae pv. tomato DC3000 and that TGA3 recruits cytokinin-activated transcription factor ARR2 to SA-responsive defense promoters through direct protein-protein interactions, it has been concluded that TGA3 provides a molecular link between the SA and the cytokinin pathways (Choi et al. 2011).

Moreover, TGA3 plays a role in the regulation of long-distance cadmium transport. The tga3-2 mutant accumulates higher amounts of cadmium in the root and lower amounts in the shoot when compared with wild-type plants (Farinati et al. 2010). Ectopically expressed TGA3 from Brassica juncea restores the transport to the shoot and confers higher cadmium tolerance. Consistently, basal and cadmium-induced transcript levels of transporters AtMRP3 and AtPDR8 are reduced in 
tga3-2. DNA binding of TGA3 is enhanced in vitro by calmodulin (Szymanski et al. 1996), but it is not yet known whether this is relevant for either of the two known functions of TGA3 or whether it plays a role in a not yet identified functional context.

So far, no information on TGA7 is yet available, so that a potential function can only be inferred from its expression pattern (Zimmermann et al. 2004). In contrast to other TGA factors, it is highly expressed in the xylem or under conditions that induce xylem differentiation.

\section{Playing in different teams: Clade II TGA factors regulate the detoxification pathway.}

The discovery that clade II TGA factors play a central role in SAR was a major breakthrough in understanding SAR establishment; still, the question on the regulation of TGA function within the SA- and 2,4-D-activated stress pathways had remained unanswered. Not only in tobacco, but also in Arabidopsis, many glutathione S-transferase and glucosyltransferase genes are induced by SA, 2,4-D, JA, and $\mathrm{H}_{2} \mathrm{O}_{2}$ (Chen and Singh 1999; Wagner et al. 2002). Induction by SA is NPR1-independent but requires clade II TGA factors (Blanco et al. 2005, 2009). In roots, TGA4 acts as a negative regulator of this response (Foley and Singh 2004). In contrast to the NPR1-dependent genes, which respond to a reducing environment after SA treatment or accumulation, the NPR1-independent genes seem to be activated through reactive oxygen species (Garreton et al. 2002). As glutathione $S$-transferases and glucosyltransferases are part of the detoxification pathway, their regulation may be viewed in this context. Along with these lines of evidence, microarray analysis after treatment of Arabidopsis plants with reactive oxidized fatty acids (e.g., phytoprostanes) revealed that TGA factors are required for the expression of $60 \%$ of the induced genes, most of them being related to detoxification, stress responses, and secondary metabolism (Mueller et al. 2008). Consistently, many safener-induced genes are controlled by clade II TGA factors but not by NPR 1 (Behringer et al. 2011). A yeast protein interaction screen yielded the GRAS protein SCL14, which functions as a coactivator of clade II TGA factors at a subset of promoters that are induced by different chemicals like SA, 2,4-D, or 2,3,5-triiodobenzoic acid. Chromatin immunoprecipitation experiments indicated that the TGA/SCL14 complex is bound to these promoters already in the noninduced state (Fode et al. 2008). Whether SCL14 is activated by redox-mediated changes like NPR1 or whether other activation steps are involved remains to be investigated (Fig. 2B).

\section{Playing in different teams: Clade II TGA transcription factors are essential activators of JA/ethylene (ET)-induced defense responses.}

Ten years after having discovered that clade II TGA factors are important for the establishment of the SA-dependent defense response SAR, it was reported that they are also required for the activation of the JA/ET-dependent defense pathway (Zander et al. 2010). Consistently, the tga2-1 tga5-1 tga6-1 mutant displays increased disease symptoms after infection with the necrotrophic fungus Botrytis cinerea. Transcriptional activation of PLANT DEFENSIN (PDF1) 1.2, a marker gene of the $\mathrm{JA} / \mathrm{ET}$ response, depends on TGA factors after application of the ET precursor 1-aminocyclopropane-carboxylic acid, either alone or in combination with JA, and after Botrytis infection (Fig. 2C). TGA6, which is redundant to TGA2 and TGA5 with respect to INA-induced $P R-1$ expression (Zhang et al. 2003), cannot support activation of $P D F 1.2$. Since SA suppresses the JA/ET pathway, TGA factors were considered to be candidates for functioning as the molecular link between the SA and the
JA/ET pathway. The analysis of the contribution of clade II TGA factors to the SA antagonism was hampered by the fact that induction of PDF 1.2 by the combined treatment with JA and ET is already compromised in the tga2-1 tga5-1 tga6-1 mutant. In order to avoid this dilemma, the tga2-1 tga5-1 tga6-1 mutant was crossed with the jinl-1 mutant, which lacks the negative regulator of the JA/ET response MYC2. The jin1-1 allele restores JA/ET-induced transcription of the $P D F 1.2$ gene in the tga2-1 tga5-1 tga6-1 background, indicating that the function of TGA factors within the JA/ET pathway is to counteract the negative effect of MYC2. Importantly, the SA antagonism is compromised in the jin1-1 tga2-1 tga5-1 tga6-1 quadruple mutant, documenting that TGA factors integrate the SA signal. Since the negative effect of SA on the JA/ET pathway is NPR1-independent (Leon-Reyes et al. 2009), other TGAinteracting proteins have to be postulated that would facilitate and modify the positive function of TGA factors. Candidates are GRX, small heat-stable oxidoreductases that transfer electrons from glutathione to oxidized cysteine residues. All 21 land plant-specific GRX (called ROXY) interact with TGA2 (Zander et al. 2011). A particularly strong candidate for mediating the SA-JA/ET cross-talk is GRX480/ROXY19, which is induced by JA and SA and interferes with $P D F 1.2$ expression when constitutively expressed in transgenic plants (Ndamukong et al. 2007; Zander et al. 2011). Thus, GRX might modulate the redox state of TGA factors or other proteins resulting in suppression of the JA/ET defense pathway.

TGA factors are not required if PDF1.2 is induced by exogenous JA in the absence of elevated ET levels. Still, inhibition of JA-induced PDF1.2 expression by SA depends on clade II TGA factors and can be suppressed by ectopically expressed GRX480/ROXY19 (Ndamukong et al. 2007; Leon-Reyes et al. 2010). Under these conditions, SA-induced expression of GRX480/ROXY19 is partially NPR1-dependent, which is also the case for the SA-JA antagonism. Up to now, there is no loss-of-function evidence for the role of GRX480/ROXY19 in regulating the cross-talk between the SA and the JA/ET defense pathway. Whether this is due to the redundancy of the GRX or another TGA-dependent mechanism exists has remained elusive.

\section{The developmental role of TGA factors: Defense responses and flower organ formation evolved from a common regulatory module.}

TGA factor PERIANTHIA (PAN) plays a role in flower development (Chuang et al. 1999). PAN is a repressor of petal formation and its mutation causes the development of one additional organ in the first two whorls. Like the other TGA factors (Ndamukong et al. 2007), PAN interacts with ROXYtype GRX ( $\mathrm{Li}$ et al. 2009). Since roxyl mutants have, on average, 2.5 petals, whereas pan has five, it can be assumed that ROXY inactivates PAN. When cysteine residue 340 in PAN is mutated into a serine, the resultant protein cannot complement the pan phenotype, indicating that PAN is only active when this cysteine is oxidized. Oxidized PAN in roxyl would lead to the strong suppression of petal number. Consistently, the pan roxyl phenotype is identical to the pan phenotype. However, direct biochemical evidence for redox modification of PAN is still missing.

Plants lacking the basic leucine-zipper transcription factors TGA9 and TGA10 have defects in male gametogenesis that are similar to those observed in roxyl roxy 2 mutants. Since TGA9 and TGA10 directly interact with ROXY proteins in yeast and in plant cell nuclei, it is suggested that the activity of TGA9 and TGA10 is controlled through ROXY-mediated modifications involving the conserved cysteine in Q1 (Fig. 1) As already observed for TGA1 and TGA4 (Lindermayr et al. 
2011), the cDNAs for TGA9 and TGA10 are unable to complement the tga9 tgal0 phenotype, so that mutations of critical cysteines could not be tested (Murmu et al. 2010).

Interestingly, PAN interacts with two NPR1-like genes, BLADE-ON-PETIOLE1 (BOP1) and BOP2 (Hepworth et al. 2005). Mutants bopl bop2 and pan share a pentamerous arrangement of first whorl floral organs. These data indicate that BOP proteins control patterning via direct interactions with TGA transcription factors, which are, in turn, connected to the action of GRX. Thus, the regulatory triad involving TGA factors, NPR-like proteins, and GRX has been duplicated, with one copy serving defense responses in leaves and the other copy functioning in the context of flower organ formation.

\section{Not yet established: Functional relevance of posttranslational modifications, protein stability, and subcellular localization of TGA factors.}

As outlined above, redox-modifications might control the activity of TGA factors, although in vivo evidence for functional relevance of such modifications is missing for members of clades I, II, and III. Protein phosphorylation constitutes another way of regulating transcription factor activity. In tobacco protein extracts, SA-induced DNA binding of tobacco TGA2.2 was sensitive to phosphatase treatment (Jupin and Chua 1996; Niggeweg et al. 2000). Further studies suggested that a casein protein kinase 2-related protein is involved in the activation of the $a s$-1-binding activity of tobacco proteins in either whole cell or nuclear extracts (Stange et al. 1997; Hidalgo et al. 2001). Taken together, these results suggest that phosphorylation of either TGA factors or interacting proteins leads to increased DNA binding. Another study demonstrated SA-induced phosphorylation at serine and threonine residues in the $\mathrm{N}$ terminus of TGA2 (Fig. 1) (Kang and Klessig 2005). However, this modification negatively regulates TGA2 DNA-binding activity. The phosphorylated sequence STDxDT is conserved in tobacco TGA2.2, hinting at a potential relevance. When expressed in transgenic Arabidopsis plants, TGA2-green fluorescent protein (GFP) and mTGA2-GFP lacking the putative phosphorylation sites activate $P R-1$ to the same levels (Kang and Klessig 2005). Still, it might be worthwhile to revisit the importance of these residues for other TGA-mediated stressregulated genes.
Moreover, it remains to be investigated whether regulation of protein turnover plays a role in the control of TGA factor activity (Pontier et al. 2002). TGA factors interact with the SA receptor proteins NPR3 and NPR4 (Fu et al. 2012; Zhang et al. 2006), which recruit interacting proteins into CUL3-Rbx1 E3 ubiquitin ligase complexes and might thus regulate SA-dependent TGA degradation. Finally, TGA2 is detected both in the cytosol and the nucleus (Cheng et al. 2009). Whether TGA2 forms already a complex with NPR1 or SCL14 in this compartment is not known. The importance of the cytosolic fraction is an issue that remains to be addressed.

\section{TGA-interacting proteins: An overview.}

As outlined in this review, several TGA-interacting proteins are known to modulate their activity. Further potential regulators have been identified in a systemic yeast two-hybrid assay, where a collection of approximately 8,000 open reading frames representing about $30 \%$ of the predicted protein-coding genes of the Arabidopsis genome were tested (The Arabidopsis Interactome Mapping Consortium 2011). This analysis included TGA factors TGA1, TGA2, TGA3, TGA5, and TGA6. The interactions with NPR1, NPR3, NPR4, and GRX ROXY1 and GRX480/ROXY19 were confirmed for at least two of the five TGA factors. Moreover, an interaction with the NPR1-interacting small protein NIMIN1 (Weigel et al. 2005) was demonstrated for TGA2 and TGA6. Not-yet-described interactions between single TGA factors and other proteins like, for example, a Myb-like helixturn-helix transcription factor (At2g38300), an E3 ligase (At2g25700), a serine threonine kinase (At3g12200) or the TFIID-related protein ENHANCED ETHYLENE RESPONSE 4 (At1g25490) were found. In yet another study, TGA5 and TGA6 were listed to interact with proteins related to the corepressor TOPLESS (Causier et al. 2011). The functional relevance of these interactions might be worthwhile investigating.

Moreover, TGA factors have been shown to interact with other transcription factors. For instance, SA-induced DOF transcription factors (Kang and Singh 2000) and the ET-inducible GCC box-binding protein AtEBP/RAP2.3 (At3g16770) (Buttner and Singh 1997) were found in protein interaction screens using TGA4 (OBF4) as a bait. In addition, TGA4 interacts with CONSTANS, a transcriptional activator of the Flowering Locus $T$ (Song et al. 2008). These factors might enhance binding of TGA transcription factors to TGACG motifs in

Table 1. Function of TGA factors

\begin{tabular}{|c|c|c|c|}
\hline TGA factors & Function $^{\mathrm{a}}$ & Interacting protein & Reference \\
\hline Clade I (TGA4) & Repression of GST6 expression in roots & & Foley and Singh 2004 \\
\hline Clade I (TGA1/4) & $\begin{array}{l}\text { Activation of basal resistance } \\
\text { Activation of defense responses elicited by the constitutively active mutant } \\
\text { R protein SNC1 }\end{array}$ & & $\begin{array}{l}\text { Kesarwani et al. } 2007 \\
\text { Shearer et al. } 2012\end{array}$ \\
\hline Clade II (TGA 2) & Negative regulation of basal $P R-1$ transcript levels & & Kesarwani et al. 2007 \\
\hline Clade II (TGA2/5) & Activation of JA/ET-induced $P D F 1.2$ expression & & Zander et al. 2010 \\
\hline Clade II (TGA2/5/6) & $\begin{array}{l}\text { Activation of } P R-1 \text { expression after INA treatment } \\
\text { Activation of early SA-induced NPR1-independent genes } \\
\text { Activation of detoxification genes } \\
\text { SA-mediated suppression of the JA/ET pathway }\end{array}$ & $\begin{array}{l}\text { NPR1 } \\
\text { SCL14 } \\
\text { GRX480/ROXY19 }\end{array}$ & $\begin{array}{l}\text { Zhang et al. } 2003 \\
\text { Blanco et al. 2005 } \\
\text { Müller et al. 2008; } \\
\text { Fode et al. 2008 } \\
\text { Ndamukong et al. 2007; } \\
\text { Zander et al. } 2010\end{array}$ \\
\hline Clade III (TGA3) & $\begin{array}{l}\text { Activation of basal } P R-1 \text { transcript levels in the absence of class II TGA factors } \\
\text { Activation of basal resistance } \\
\text { Regulation of long-distance cadmium transport } \\
\text { Enhancement of SA-dependent defense responses after cytokinin treatment }\end{array}$ & ARR2 & $\begin{array}{l}\text { Kesarwani et al. } 2007 \\
\text { Kesarwani et al. } 2007 \\
\text { Farinati et al. } 2010 \\
\text { Choi et al. } 2011\end{array}$ \\
\hline PAN & Repressor of formation of a fifth petal & ROXY1 & Chuang et al. 1999 \\
\hline Clade IV (TGA9/10) & Anther development & ROXY1/2 & Murmu et al. 2010 \\
\hline
\end{tabular}

${ }^{\mathrm{a}}$ Functions as determined by loss of function analysis of single, double, and triple Arabidopsis mutants are summarized. JA = jasmonic acid, ET $=$ ethylene, and $\mathrm{SA}=$ salicylic acid. 
their vicinity, in which they might either activate or repress transcription. Several reports have documented a stimulating activity of the $a s-1$ element in different promoter contexts. For instance, although the isolated $a s-1$ element cannot mediate light-responsive gene expression, it is necessary to support light-induced gene expression from a chimeric promoter consisting of four light-responsive cis elements fused to the $\mathrm{CaMV}$ $35 S$ core promoter (Lam and Chua 1990). Likewise, although the $a s-1$ element cannot mediate ET-induced gene expression, it is important for ET-induced gene expression in the context of the PDF1.2 promoter (Zander et al. 2010). In the context of the CYP81D11 promoter, it is essential for JA induction (Koster et al. 2012). Being the target of SA signaling, TGA factors can thus connect the SA pathway with the JA/ET pathway. It can be assumed that more examples for such interplays will be discovered when further exploring the function of TGA factors.

In conclusion, members of the TGA family of transcription factors regulate gene expression in response to many environmental and developmental cues (Table 1). Depending on the promoter context they can recruit different transcriptional coactivators, such as SA-activated NPR1 or SCL14. Moreover, they can enhance the activity of other transcription factors at a variety of promoters. All TGA factors interact with ROXYtype GRX and all 21 Arabidopsis ROXY interact with Arabidopsis TGA2. This interaction and the interaction with NPRtype proteins have been conserved after numerous rounds of gene duplications that have separated NPR, TGA, and ROXY regulatory modules into those that are involved in flower development and those regulating defense responses. It will be fascinating to elucidate whether TGA factors are direct substrates of ROXY or whether TGA interacting proteins are subject to ROXY-mediated redox-modulations, or both.

\section{LITERATURE CITED}

The Arabidopsis Interactome Mapping Consortium. 2011. Evidence for network evolution in an Arabidopsis interactome map. Science 333:601-607.

Behringer, C., Bartsch, K., and Schaller, A. 2011. Safeners recruit multiple signalling pathways for the orchestrated induction of the cellular xenobiotic detoxification machinery in Arabidopsis. Plant Cell Environ. 34:970-1985

Benfey, P. N., Ren, L., and Chua, N. H. 1990. Tissue-specific expression from CaMV 35S enhancer subdomains in early stages of plant development. EMBO (Eur. Mol. Biol. Organ.) J. 9:1677-1684.

Blanco, F., Garreton, V., Frey, N., Dominguez, C., Perez-Acle, T., Van der Straeten, D., Jordana, X., and Holuigue, L. 2005. Identification of NPR1-dependent and independent genes early induced by salicylic acid treatment in Arabidopsis. Plant Mol. Biol. 59:927-944.

Blanco, F., Salinas, P., Cecchini, N.M., Jordana, X., Van Hummelen, P., Alvarez, M. E., and Holuigue, L. 2009. Early genomic responses to salicylic acid in Arabidopsis. Plant Mol. Biol. 70:79-102.

Boyle, P., Le Su, E., Rochon, A., Shearer, H. L., Murmu, J., Chu, J. Y., Fobert, P. R., and Despres, C. 2009. The BTB/POZ domain of the Arabidopsis disease resistance protein NPR1 interacts with the repression domain of TGA2 to negate its function. Plant Cell 21:3700-3713.

Buttner, M., and Singh, K. B. 1997. Arabidopsis thaliana ethylene-responsive element binding protein (AtEBP), an ethylene-inducible, GCC box DNA-binding protein interacts with an ocs element binding protein. Proc. Natl. Acad. Sci. U.S.A. 94:5961-5966.

Causier, B., Ashworth, M., Guo, W., and Davies, B. 2012. The TOPLESS interactome: A framework for gene repression in Arabidopsis. Plant Physiol. 158:423-438.

Chen, W., and Singh, K. B. 1999. The auxin, hydrogen peroxide and salicylic acid induced expression of the Arabidopsis GST6 promoter is mediated in part by an ocs element. Plant J. 19:667-677.

Cheng, Y. T., Germain, H., Wiermer, M., Bi, D., Xu, F., Garcia, A. V., Wirthmueller, L., Despres, C., Parker, J. E., Zhang, Y., and Li, X. 2009. Nuclear pore complex component MOS7/Nup88 is required for innate immunity and nuclear accumulation of defense regulators in Arabidopsis. Plant Cell 21:2503-2516.

Choi, J., Huh, S. U., Kojima, M., Sakakibara, H., Paek, K. H., and Hwang,
I. 2011. The cytokinin-activated transcription factor ARR2 promotes plant immunity via TGA3/NPR1-dependent salicylic acid signaling in Arabidopsis. Dev. Cell 19:284-295.

Chuang, C. F., Running, M. P., Williams, R. W., and Meyerowitz, E. M. 1999. The PERIANTHIA gene encodes a bZIP protein involved in the determination of floral organ number in Arabidopsis thaliana. Genes Dev. 13:334-344.

Despres, C., DeLong, C., Glaze, S., Liu, E., and Fobert, P. R. 2000. The Arabidopsis NPR1/NIM1 protein enhances the DNA binding activity of a subgroup of the TGA family of bZIP transcription factors. Plant Cell 12:279-290.

Despres, C., Chubak, C., Rochon, A., Clark, R., Bethune, T., Desveaux, D., and Fobert, P. R. 2003. The Arabidopsis NPR1 disease resistance protein is a novel cofactor that confers redox regulation of DNA binding activity to the basic domain/leucine zipper transcription factor TGA1. Plant Cell 15:2181-2191.

Ellenberger, T. E., Brandl, C. J., Struhl, K., and Harrison, S. C. 1992. The GCN4 basic region leucine zipper binds DNA as a dimer of uninterrupted alpha helices: Crystal structure of the protein-DNA complex. Cell 71:1223-1237.

Ellis, J. G., Tokuhisa, J. G., Llewellyn, D. J., Bouchez, D., Singh, K., Dennis, E. S., and Peacock, W. J. 1993. Does the ocs-element occur as a functional component of the promoters of plant genes? Plant J. 4:433443.

Fan, W., and Dong, X. 2002. In vivo interaction between NPR1 and transcription factor TGA2 leads to salicylic acid-mediated gene activation in Arabidopsis. Plant Cell 14:1377-1389.

Farabaugh, P. J. 1978. Sequence of the lacI gene. Nature 274:765-769.

Farinati, S., DalCorso, G., Varotto, S., and Furini, A. 2010. The Brassica juncea $\mathrm{BjCdR} 15$, an ortholog of Arabidopsis TGA3, is a regulator of cadmium uptake, transport and accumulation in shoots and confers cadmium tolerance in transgenic plants. New Phytol. 185:964-978.

Fode, B., Siemsen, T., Thurow, C., Weigel, R., and Gatz, C. 2008. The Arabidopsis GRAS protein SCL14 interacts with class II TGA transcription factors and is essential for the activation of stress-inducible promoters. Plant Cell 20:3122-3135.

Foley, R. C., and Singh, K. B. 2004. TGA5 acts as a positive and TGA4 acts as a negative regulator of ocs element activity in Arabidopsis roots in response to defence signals. FEBS Lett. 563:141-145.

Fu, Z. Q., Yan, S., Saleh, A., Wang, W., Ruble, J., Oka, N., Mohan, R., Spoel, S. H., Tada, Y., Zheng, N., and Dong, X. 2012. NPR3 and NPR4 are receptors for the immune signal salicylic acid in plants. Nature 486:228-232.

Garreton, V., Carpinelli, J., Jordana, X., and Holuigue, L. 2002. The as-1 promoter element is an oxidative stress-responsive element and salicylic acid activates it via oxidative species. Plant Physiol. 130:1516-1526.

Hepworth, S. R., Zhang, Y., McKim, S., Li, X., and Haughn, G. W. 2005. BLADE-ON-PETIOLE-dependent signaling controls leaf and floral patterning in Arabidopsis. Plant Cell 17:1434-1448.

Hidalgo, P., Garreton, V., Berrios, C. G., Ojeda, H., Jordana, X., and Holuigue, L. 2001. A nuclear casein kinase 2 activity is involved in early events of transcriptional activation induced by salicylic acid in tobacco. Plant Physiol. 125:396-405.

Hollenberg, S. M., Weinberger, C., Ong, E. S., Cerelli, G., Oro, A., Lebo, R., Thompson, E. B., Rosenfeld, M. G., and Evans, R.M. 1985. Primary structure and expression of a functional human glucocorticoid receptor cDNA. Nature 318:635-641.

Izawa, T., Foster, R., and Chua, N. H. 1993. Plant bZIP protein DNA binding specificity. J. Mol. Biol. 230:1131-1144.

Jakoby, M., Weisshaar, B., Droge-Laser, W., Vicente-Carbajosa, J. Tiedemann, J., Kroj, T., and Parcy, F. 2002. bZIP transcription factors in Arabidopsis. Trends Plant Sci. 7:106-111.

Johnson, C., Glover, G., and Arias, J. 2001. Regulation of DNA binding and trans-activation by a xenobiotic stress-activated plant transcription factor. J. Biol. Chem. 276:172-178

Johnson, C., Boden, E., and Arias, J. 2003. Salicylic acid and NPR1 induce the recruitment of trans-activating TGA factors to a defense gene promoter in Arabidopsis. Plant Cell 15:1846-1858.

Johnson, C., Mhatre, A., and Arias, J. 2008. NPR1 preferentially binds to the DNA-inactive form of Arabidopsis TGA2. Biochem. Biophys. Act. 1779:583-589.

Jupin, I., and Chua, N. H. 1996. Activation of the CaMV as-1 cis-element by salicylic acid: Differential DNA-binding of a factor related to TGA1a. EMBO (Eur. Mol. Biol. Organ.) J. 15:5679-5689.

Kadonaga, J. T., Carner, K. R., Masiarz, F. R., and Tjian, R. 1987. Isolation of cDNA encoding transcription factor $\mathrm{Sp} 1$ and functional analysis of the DNA binding domain. Cell 51:1079-1090.

Kang, H. G. and Klessig, D. F. 2005. Salicylic acid-inducible Arabidopsis CK2-like activity phosphorylates TGA2. Plant Mol. Biol:57, 541-557.

Kang, H. G., and Singh, K. B. 2000. Characterization of salicylic acid- 
responsive Arabidopsis Dof domain proteins: Overexpression of OBP3 leads to growth defects. Plant J. 21:329-339.

Katagiri, F., Lam, E., and Chua, N. H. 1989. Two tobacco DNA-binding proteins with homology to the nuclear factor CREB. Nature 340:727730

Katagiri, F., Seipel, K., and Chua, N. H. 1992. Identification of a novel dimer stabilization region in a plant bZIP transcription activator. Mol. Cell. Biol. 12:4809-4816.

Kegler, C., Lenk, I., Krawczyk, S., Scholz, R., and Gatz, C. 2004. Functional characterization of tobacco transcription factor TGA2.1. Plant Mol. Biol. 55:153-164.

Kesarwani, M., Yoo, J., and Dong, X. 2007. Genetic interactions of TGA transcription factors in the regulation of pathogenesis-related genes and disease resistance in Arabidopsis. Plant Physiol. 144:336-346.

Klinedinst, S., Pascuzzi, P., Redman, J., Desai, M., and Arias, J. 2000. A xenobiotic-stress-activated transcription factor and its cognate target genes are preferentially expressed in root tip meristems. Plant Mol. Biol. 42:679-688.

Koster, J., Thurow, C., Kruse, K., Meier, A., Iven, T., Feussner, I., and Gatz, C. 2012. Xenobiotic- and jasmonic acid-inducible signal transduction pathways have become interdependent at the Arabidopsis CYP81D11 promoter. Plant Physiol. 159:391-402.

Lam, E., and Chua, N. H. 1990. GT-1 binding site confers light responsive expression in transgenic tobacco. Science 248:471-474.

Lam, E., and Lam, Y. K. 1995. Binding site requirements and differential representation of TGF factors in nuclear ASF-1 activity. Nucleic Acids Res. 23:3778-3785.

Lam, E., Benfey, P. N., Gilmartin, P. M., Fang, R. X., and Chua, N. H. 1989. Site-specific mutations alter in vitro factor binding and change promoter expression pattern in transgenic plants. Proc. Natl. Acad. Sci. U.S.A. 86:7890-7894.

Landschulz, W. H., Johnson, P. F., and McKnight, S. L. 1988. The leucine zipper: A hypothetical structure common to a new class of DNA binding proteins. Science 240:1759-1764.

Laughon, A., and Gesteland, R. F. 1984. Primary structure of the Saccharomyces cerevisiae GAL4 gene. Mol. Cell. Biol. 4:260-267.

Lebel, E., Heifetz, P., Thorne, L., Uknes, S., Ryals, J., and Ward, E. 1998. Functional analysis of regulatory sequences controlling $P R-1$ gene expression in Arabidopsis. Plant J. 16:223-233.

Leon-Reyes, A., Spoel, S. H., De Lange, E. S., Abe, H., Kobayashi, M., Tsuda, S., Millenaar, F. F., Welschen, R. A., Ritsema, T., and Pieterse, C. M. 2009. Ethylene modulates the role of NONEXPRESSOR OF PATHOGENESIS-RELATED GENES1 in cross talk between salicylate and jasmonate signaling. Plant Physiol. 149:1797-1809.

Leon-Reyes, A., Du, Y., Koornneef, A., Proietti, S., Körbes, A. P., Memelink, J., Pieterse, C. M., and Ritsema, T. 2010. Ethylene signaling renders the jasmonate response of Arabidopsis insensitive to future suppression by salicylic acid. Mol. Plant-Microbe Interact. 23:187-197.

Li, S., Lauri, A., Ziemann, M., Busch, A., Bhave, M., and Zachgo, S 2009. Nuclear activity of ROXY1, a glutaredoxin interacting with TGA factors, is required for petal development in Arabidopsis thaliana. Plant Cell 21:429-441.

Li, X., Song, Y., Century, K., Straight, S., Ronald, P., Dong, X., Lassner, M., and Zhang, Y. 2001. A fast neutron deletion mutagenesis-based reverse genetics system for plants. Plant J. 27:235-242.

Lindermayr, C., Sell, S., Muller, B., Leister, D., and Durner, J. 2010. Redox regulation of the NPR1-TGA1 system of Arabidopsis thaliana by nitric oxide. Plant Cell 22:2894-2907.

Liu, X., and Lam, E. 1994. Two binding sites for the plant transcription factor ASF-1 can respond to auxin treatments in transgenic tobacco. J. Biol. Chem. 269:668-675.

Lorenzo, O., Chico, J. M., Sánchez-Serrano, J. J., and Solano, R. 2004 JASMONATE-INSENSITIVE1 encodes a MYC transcription factor essential to discriminate between different jasmonate-regulated defense responses in Arabidopsis. Plant Cell 16:1938-1350.

Mou, Z., Fan, W., and Dong, X. 2003. Inducers of plant systemic acquired resistance regulate NPR1 function through redox changes. Cell 113:935-944.

Mueller, S., Hilbert, B., Dueckershoff, K., Roitsch, T., Krischke, M., Mueller, M. J., and Berger, S. 2008. General detoxification and stress responses are mediated by oxidized lipids through TGA transcription factors in Arabidopsis. Plant Cell 20:768-785.

Murmu, J., Bush, M. J., DeLong, C., Li, S., Xu, M., Khan, M., Malcolmson, C., Fobert, P. R., Zachgo, S., and Hepworth, S.R. 2010. Arabidopsis basic leucine-zipper transcription factors TGA9 and TGA10 interact with floral glutaredoxins ROXY1 and ROXY2 and are redundantly required for anther development. Plant Physiol. 154:1492-1504.

Ndamukong, I., Abdallat, A. A., Thurow, C., Fode, B., Zander, M., Weigel, R., and Gatz, C. 2007. SA-inducible Arabidopsis glutaredoxin interacts with TGA factors and suppresses JA-responsive PDF1.2 transcription.
Plant J. 50:128-139.

Neuhaus, G., Neuhaus-Url, G., Katagiri, F., Seipel, K., and Chua, N. H. 1994. Tissue-specific expression of as-1 in transgenic tobacco. Plant Cell 6:827-834.

Niggeweg, R., Thurow, C., Kegler, C., and Gatz, C. 2000. Tobacco transcription factor TGA2.2 is the main component of $a s$-1-binding factor ASF-1 and is involved in salicylic acid- and auxin-inducible expression of as-1-containing target promoters. J. Biol. Chem. 275:19897-19905.

Pape, S., Thurow, C., and Gatz, C. 2010. The Arabidopsis thaliana PR-1 promoter contains multiple integration sites for the co-activator NPR1 and the repressor SNI1. Plant Physiol. 154:1805-1818.

Pascuzzi, P., Hamilton, D., Bodily, K., and Arias, J. 1998. Auxin-induced stress potentiates trans-activation by a conserved plant basic/leucinezipper factor. J. Biol. Chem. 273:26631-26637.

Penn, M. D., Thireos, G., and Greer, H. 1984. Temporal analysis of general control of amino acid biosynthesis in Saccharomyces cerevisiae: Role of positive regulatory genes in initiation and maintenance of mRNA derepression. Mol. Cell. Biol. 4:520-528.

Pontier, D., Privat, I., Trifa, Y., Zhou, J. M., Klessig, D. F., and Lam, E. 2002. Differential regulation of TGA transcription factors by post-transcriptional control. Plant J. 32:641-653.

Qin, X. F., Holuigue, L., Horvath, D. M., and Chua, N. H. 1994. Immediate early transcription activation by salicylic acid via the cauliflower mosaic virus as-1 element. Plant Cell 6:863-874.

Rieping, M., Fritz, M., Prat, S., and Gatz, C. 1994. A dominant negative mutant of PG13 suppresses transcription from a cauliflower mosaic virus $35 \mathrm{~S}$ truncated promoter in transgenic tobacco plants. Plant Cell 6:1087-1098.

Rochon, A., Boyle, P., Wignes, T., Fobert, P. R., and Despres, C. 2006. The coactivator function of Arabidopsis NPR1 requires the core of its $\mathrm{BTB} / \mathrm{POZ}$ domain and the oxidation of C-terminal cysteines. Plant Cell 18:3670-3685.

Ryder, K., Lau, L. F., and Nathans, D. 1988. A gene activated by growth factors is related to the oncogene v-jun. Proc. Natl. Acad. Sci. U.S.A. 85:1487-1491.

Schindler, U., Beckmann, H., and Cashmore, A. R. 1992. TGA1 and G-box binding factors: Two distinct classes of Arabidopsis leucine zipper proteins compete for the G-box-like element TGACGTGG. Plant Cell 4:1309-1319

Shearer, H. L., Cheng, Y. T., Wang, L., Liu, J., Boyle, P., Després, C., Zhang, Y., Li, X., Fobert, P. R. 2012. Arabidopsis clade I TGA transcription factors regulate plant defenses in an NPR1-independent fashion. Mol. Plant-Microbe Interact. 25:1459-1468.

Singh, H., LeBowitz, J. H., Baldwin, A. S. Jr., and Sharp, P. A. 1988. Molecular cloning of an enhancer binding protein: Isolation by screening of an expression library with a recognition site DNA. Cell 12:415-423.

Song, Y. H., Song, N. Y., Shin, S. Y., Kim, H. J., Yun, D. J., Lim, C. O., Lee, S. Y., Kang, K. Y., and Hong, J. C. 2008. Isolation of CONSTANS as a TGA4/OBF4 interacting protein. Mol. Cells 25:559-565.

Spoel, S. H., Mou, Z., Tada, Y., Spivey, N. W., Genschik, P., and Dong, X. 2009. Proteasome-mediated turnover of the transcription coactivato NPR1 plays dual roles in regulating plant immunity. Cell 137:860-872.

Stange, C., Ramirez, I., Gomez, I., Jordana, X., and Holuigue, L. 1997. Phosphorylation of nuclear proteins directs binding to salicylic acidresponsive elements. Plant J. 11:1315-1324.

Szymanski, D. B., Liao, B., and Zielinski, R. E. 1996. Calmodulin isoforms differentially enhance the binding of cauliflower nuclear proteins and recombinant TGA3 to a region derived from the Arabidopsis Cam-3 promoter. Plant Cell 8:1069-1077.

van der Krol, A. R., and Chua, N. H. 1991. The basic domain of plant bZIP proteins facilitates import of a reporter protein into plant nuclei. Plant Cell 3:667-675.

van der Zaal, E. J., Droog, F. N., Boot, C. J., Hensgens, L. A., Hoge, J. H., Schilperoort, R. A., and Libbenga, K. R. 1991. Promoters of auxininduced genes from tobacco can lead to auxin-inducible and root tipspecific expression. Plant Mol. Biol. 16:983-998.

van der Zaal, B. J., Droog, F. N., Pieterse, F. J., and Hooykaas, P. J. 1996. Auxin-sensitive elements from promoters of tobacco GST genes and a consensus as-1-like element differ only in relative strength. Plant Physiol. 110:79-88

Wagner, U., Edwards, R., Dixon, D. P., and Mauch, F. 2002. Probing the diversity of the Arabidopsis glutathione $S$-transferase gene family. Plant Mol. Biol. 49:515-532.

Wang, D., Amornsiripanitch, N., and Dong, X. 2006. A genomic approach to identify regulatory nodes in the transcriptional network of systemic acquired resistance in plants. PLoS Path. 2:e123. Published online.

Weigel, R. R., Pfitzner, U. M., and Gatz, C. 2005. Interaction of NIMIN1 with NPR1 modulates PR gene expression in Arabidopsis. Plant Cell 17:1279-1291.

Wu, Y., Zhang, D., Chu, J. Y., Boyle, P., Wang, Y., Brindle, I. D., De Luca, 
V., and Despres, C. 2012. The Arabidopsis NPR1 protein is a receptor for the plant defense hormone salicylic acid. Cell Rep. 1:639-647.

Xiang, C., Miao, Z. H., and Lam, E. 1996. Coordinated activation of as-1type elements and a tobacco glutathione $S$-transferase gene by auxins, salicylic acid, methyl-jasmonate and hydrogen peroxide. Plant Mol. Biol. 32:415-426.

Zander, M., La Camera, S., Lamotte, O., Metraux, J. P. and Gatz, C. 2010 Arabidopsis thaliana class-II TGA transcription factors are essential activators of jasmonic acid/ethylene-induced defense responses. Plant J. 61:200-210.

Zander, M., Chen, S., Imkampe, J., Thurow, C., and Gatz, C. 2012. Repression of the Arabidopsis thaliana jasmonic acid/ethylene-induced defense pathway by TGA-interacting glutaredoxins depends on their C-terminal ALWL motif. Mol. Plant 5:831-840.

Zhang, B., and Singh, K. B. 1994. ocs element promoter sequences are activated by auxin and salicylic acid in Arabidopsis. Proc. Natl. Acad. Sci. U.S.A. 91:2507-2511.

Zhang, Y., Fan, W., Kinkema, M., Li, X., and Dong, X. 1999. Interaction of NPR1 with basic leucine zipper protein transcription factors that bind sequences required for salicylic acid induction of the $P R-1$ gene. Proc. Natl. Acad. Sci. U.S.A. 96:6523-6528.

Zhang, Y., Tessaro, M.J., Lassner, M., and Li, X. 2003. Knockout analysis of Arabidopsis transcription factors TGA2, TGA5, and TGA6 reveals their redundant and essential roles in systemic acquired resistance. Plant Cell 15:2647-2653.

Zhang, Y., Cheng, Y. T., Qu, N., Zhao, Q., Bi, D., and Li, X. 2006. Negative regulation of defense responses in Arabidopsis by two NPR1 paralogs. Plant J. 48:647-656.

Zhou, J.-M., Trifa, Y., Silva, H., Pontier, D., Lam, E., Shah, J., and Klessig, D. F. 2000. NPR1 differentially interacts with members of the TGA/OBF family of transcription factors that bind an element of the $P R-1$ gene required for induction by salicylic acid. Mol. Plant-Microbe Interact. 13:191-202.

Zimmermann, P., Hirsch-Hoffmann, M., Hennig, L. and Gruissem, W. 2004 GENEVESTIGATOR. Arabidopsis microarray database and analysis toolbox. Plant Physiol. 136:2621-2632. 